\title{
Source rock analysis, thermal maturation and hydrocarbon generation using rock-eval pyrolysis in parts of Krishna-Godavari basin, India: a case study
}

\author{
K. Ramachandran • Vinay Babu • Bijaya K. Behera • \\ T. Harinarayana
}

Received: 15 June 2012/ Accepted: 23 October 2012/Published online: 4 November 2012

(C) The Author(s) 2012. This article is published with open access at Springerlink.com

\begin{abstract}
Among the various techniques available, the geochemical technique involving total organic carbon content (TOC) and rock-eval pyrolysis are regularly being used in hydrocarbon exploration. They provide information on the kerogen type, sedimentary environment, effective source rock identification and its thermal maturity. In the present study, core samples from three deep boreholes in Krishna-Godavari basin are analyzed to understand the hydrocarbon scenario in parts of the basin. Based on TOC and rock-eval pyrolysis significantly thick, indigenous immature to early mature gas-prone shales from borehole GERMI \#1; while non-indigenous, early mature to mature oil and gas-prone shales from borehole GERMI \#2 and GERMI \#3. Interpreted hydrocarbon typing is supported by well-testing analysis. Break in vitrinite reflectance $\left(R_{\mathrm{o}}\right)$ profile is also observed; indicative of significant erosion during Late Cretaceous sedimentation due to tectonic uplift during this time and associated high heat flow causes for thermal maturation of overlying units.
\end{abstract}

Keywords Source $\cdot$ Rock potential $\cdot$ Rock-eval pyrolysis · Total organic carbon $\cdot$ Krishna-Godavari Basin

K. Ramachandran

Petroleum Research Wing, Gujarat Energy Research and

Management Institute, Gandhinagar, Gujarat, India

V. Babu $(\bowtie) \cdot$ B. K. Behera

School of Petroleum Technology, Pandit Deendayal Petroleum

University, Gandhinagar, Gujarat, India

e-mail: vinthegreat84@gmail.com

URL: http://www.spt.pdpu.ac.in/ri_vinaybabu.html

T. Harinarayana

Gujarat Energy Research and Management Institute,

Gandhinagar, Gujarat, India

\section{Introduction}

There are three major sedimentary basins along the east cost of India, namely, the Cauvery, the Krishna-Godavari and Mahanadi. Among three sedimentary basins, KrishnaGodavari (KG) basin located between the major rivers Krishna and the Godavari and also extending offshore, has attracted many oil and gas companies due to its richness in hydrocarbon potential. The location map of exploration block KG-OSN-2001/3 in Krishna-Godavari basin along with the boreholes is shown in Fig. 1. This block comprises an area of $1,850 \mathrm{~km}^{2}$ in NE-SW trending KrishnaGodavari (KG) Basin and is located on the eastern coast of the Andhra Pradesh, India (Tidar and Budi 2005). It represents typical divergent passive margin of east coast. It is a proved petroliferous basin (Rai et al. 1998; Biswas 1998) with an aerial extent of $15,000 \mathrm{~km}^{2}$ on land and about $25,000 \mathrm{~km}^{2}$ of adjoining offshore area up to $1,000 \mathrm{~m}$ isobaths line (Kumar 1983). The detailed general geology and tectonics of the basin has already been described (Gupta 2006; Rao 2001). The well-known major Godavari rift extends both onland and offshore. On land, it is exposed near Wardha towards the north western direction and extends to the sea in the south east direction. In the seaward portion, it crosses the shelf and enters the deep sea. The generalized stratigraphy of Krishna-Godavari basin is shown in Fig. 3. It contains the rocks from Cretaceous to Tertiary, different rock types are sand, sandstone, mudstone, shale, clay, siltstone, silt, volcanic and limestone (IHS 2005). The Godavari Graben is filled with a maximum of 2.8-km-thick Early Gondwana (Late Permian to Early Triassic) sediments (Kaila et al. 2005). In between these horst structures, three sub-basins are, namely, Krishna, West Godavari and the East Godavari. The basin is dominated by deltaic plains near the sea-land boundary. From

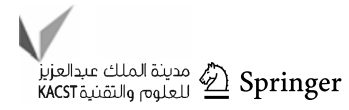




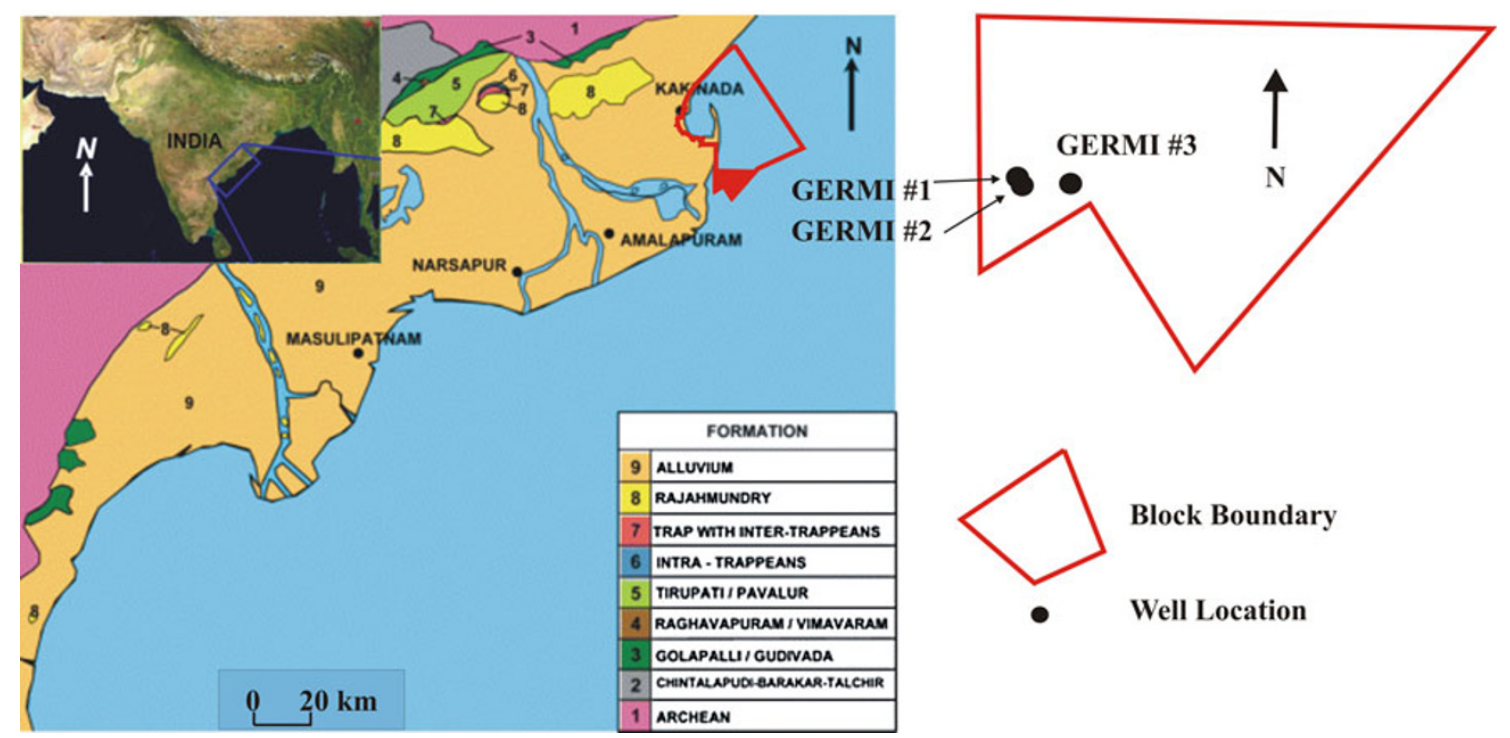

Fig. 1 Simplified geologic map of the area and location of wells (GERMI \#1, GERMI \#2 and GERMI \#3) in Krishna-Godavari basin after (Gupta 2006)

the study of gravity anomalies, three basins have been characterized separated by the two basement horsts namely, Bapatla and Tanuku (Venkatarangan and Ray 1993; Murthy et al. 1995; Subrahmanyam et al. 2008; Murthy et al. 2010). The basin has significant hydrocarbon potential both in the Tertiary delta as well as in the channel-levee-overbank play types in the deepwaters (Saxena et al. 2011).

Three important geological units of $\mathrm{KG}$ basin are Northern graben, the central Horst and several sub-basins (Fig. 2). Unlike other basins on the east coast of India, the $\mathrm{KG}$ basin is considered to be unique in nature as it is reported that hydrocarbon potential identified in almost all the stratigraphic formations. For example, Triassic Mandapeta formation, Late Jurassic Gollapalli formation, Early Cretaceous Raghavapuram formation, Late Cretaceous Tirupati formation, Eocene Pasarlapudi formation, Eocene-Oligocene-Miocene Matsyapuri formation/Ravva formation and Pliocene Godavari clay formations, all know to be rich in hydrocarbons (Fig. 3). More prominently, the Ravva structure and Godavari formation of Paleocene period are all known to contain significant hydrocarbon potential (Govindan 1984) and thus the basin has emerged as one of the most promising area for exploration. These reservoirs have been charged at different maturity levels of corresponding sources within different stratigraphic sequences (Saxena et al. 2011).

In this region, recently $15 \mathrm{TCF}$ of gas initially in-place (GIIP) and 38,000 barrels oil is proved through exploration drilling process (IHS 2005). Although much of the area on the land has been investigated in detail, the offshore region is relatively poorly investigated due to high cost of drilling in offshore region. In view of this, it is necessary to make a detailed and careful analysis of the meagerly available data from the offshore boreholes. In view of the above, present study is aimed to make a detailed analysis of a few borehole core samples from three deep offshore wells using the well-known TOC and rock-eval pyrolysis tools.

\section{Sampling and methodology}

The data acquired for the present study are mainly related to three offshore deep boreholes of KG basin (Table 1). The appropriate location of the wells namely GERMI \#1, GERMI \#2 and GERMI \#3 are presented in Fig. 4. From these three boreholes, a number of 38 drillcore samples have been subjected to detailed analysis for TOC and rockeval pyrolysis. Rock-eval pyrolysis study of samples has been carried out in ACS lab (Tidar and Budi 2005). In general, samples with TOC values greater than $0.5 \%$ for shales and $0.3 \%$ for carbonates are considered suitable for rock-eval pyrolysis. Rock samples are pyrolyzed using Rock-Eval 6 apparatus at ACS core laboratory. This technique evaluates oil and gas potential, the generation, thermal maturity and identifies organic matter type (Peters 1986; Tissot and Welte 1978). This instrument uses a ramped temperature pyrolysis technique, whereby a small amount of sample (70-100 mg) is heated in an inert atmosphere (helium or nitrogen) and also combusted with air to obtain several key geochemical parameters relating to the hydrocarbon potential of the rock such as the total organic carbon (TOC), type or quality of organic matter and maturity level (Peters 1986; Lafargue et al. 1998; Behar et al. 2001). 
Fig. 2 Tectonic map of

Krishna-Godavari basin after (Gupta 2006). All the wells are in western Godavari sub-basin

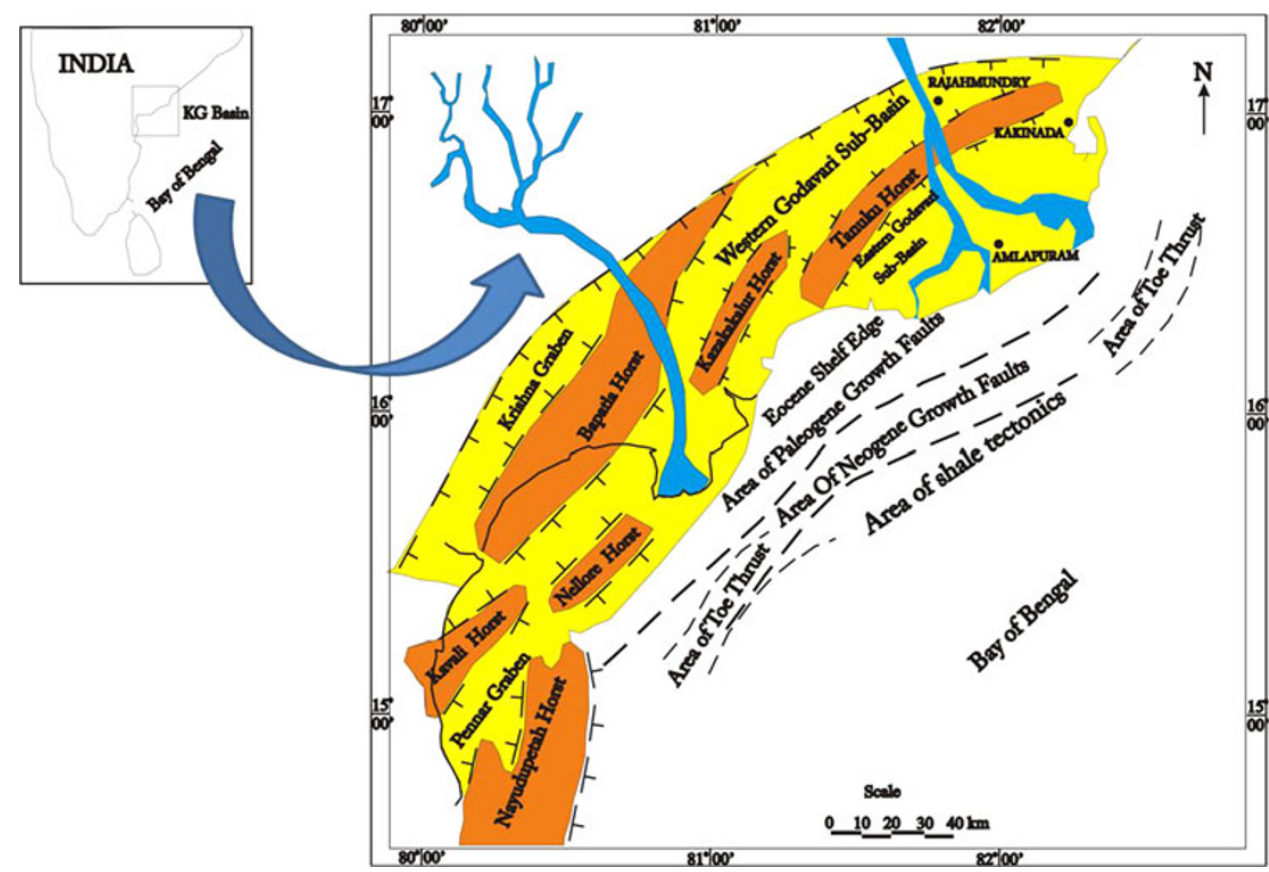

Fig. 3 General stratigraphy of the basin along with different oil and gas pools (Gupta 2006)

\section{Results and discussions}

Depth ranges of well bore ranges from 2,300 to $5,100 \mathrm{~m}$ covering the Early Cretaceous, Paleocene and Early Eocene. Figure 5 shows the relationship between hydrogen index $(\mathrm{HI})$ and $T_{\max }$ values in these sediments from
GERMI \#1, 2 and 3 in KG Basin. Thermal alteration pathways of organic matter Types-I, -II, and -III are indicated by dashed lines. Solid lines indicate approximate vitrinite reflectance values (not measured) that define the "oil window". The geochemical results are plotted and also interpreted on hydrocarbon generation using hydrogen 
Table 1 Measured rock-eval pyrolysis and additional derived parameters

\begin{tabular}{|c|c|c|c|c|c|c|c|c|c|c|c|c|c|}
\hline \multirow[t]{2}{*}{ Depth } & \multirow[t]{2}{*}{$S_{1}$} & \multirow[t]{2}{*}{$S_{2}$} & \multirow[t]{2}{*}{$S_{3}$} & \multirow[t]{2}{*}{$S_{2} / S_{3}$} & \multirow[t]{2}{*}{ PI } & \multirow[t]{2}{*}{$S_{1}+S_{2}$} & \multirow[t]{2}{*}{ OI } & \multirow[t]{2}{*}{$T_{\max }$} & \multirow[t]{2}{*}{ TOC } & \multirow[t]{2}{*}{ HI } & \multicolumn{3}{|l|}{$R_{\mathrm{o}}$} \\
\hline & & & & & & & & & & & $\begin{array}{l}\text { Caved } \\
\text { population }\end{array}$ & Indigenous & $\begin{array}{l}\text { Oxidized } \\
\text { population }\end{array}$ \\
\hline 2,325 & 0.25 & 4.62 & 5.16 & 0.9 & 0.05 & 4.87 & 199.23 & 430 & 2.59 & 178.38 & nd & 0.5219 & nd \\
\hline 2,420 & 0.19 & 2.8 & 2.21 & 1.27 & 0.06 & 2.99 & 134.76 & 431 & 1.64 & 170.73 & nd & 0.5342 & nd \\
\hline 2,545 & 0.18 & 2.22 & 2.43 & 0.91 & 0.08 & 2.4 & 139.66 & 432 & 1.74 & 127.59 & nd & 0.5533 & nd \\
\hline 2,672 & 0.06 & 0.03 & 0.78 & 0.04 & 0.67 & 0.09 & 268.97 & - & 0.29 & 10.34 & $\mathrm{nr}$ & $\mathrm{nr}$ & $\mathrm{nr}$ \\
\hline 3,492 & 0.2 & 1.09 & 0.64 & 1.7 & 0.16 & 1.29 & 43.24 & 450 & 1.48 & 73.65 & nd & 0.9767 & nd \\
\hline 3,502 & 0.25 & 0.81 & 1.19 & 0.68 & 0.24 & 1.06 & 97.54 & 453 & 1.22 & 66.39 & nd & 0.962 & 1.387 \\
\hline 3,657 & 0.25 & 0.46 & 0.72 & 0.64 & 0.35 & 0.71 & 54.14 & 456 & 1.33 & 34.59 & nd & 0.968 & nd \\
\hline 3,815 & $\mathrm{nr}$ & $\mathrm{nr}$ & $\mathrm{nr}$ & $\mathrm{nr}$ & $\mathrm{nr}$ & $\mathrm{nr}$ & $\mathrm{nr}$ & $\mathrm{nr}$ & $\mathrm{nr}$ & $\mathrm{nr}$ & 0.436 & 1.06 & 2.53 \\
\hline 3,820 & 0.37 & 1.43 & 0.7 & 2.04 & 0.21 & 1.8 & 37.63 & 436 & 1.86 & 76.88 & nd & nd & nd \\
\hline $3,885.5$ & 0.27 & 0.88 & 0.78 & 1.13 & 0.23 & 1.15 & 58.21 & 395 & 1.34 & 65.67 & nd & 0.9669 & 1.325 \\
\hline 3,890 & 0.37 & 1.48 & 0.97 & 1.53 & 0.2 & 1.85 & 56.73 & 430 & 1.71 & 86.55 & nd & 1.06 & nd \\
\hline 3,945 & 0.15 & 0.35 & 0.81 & 0.43 & 0.3 & 0.5 & 45 & 365 & 1.8 & 19.44 & nd & 1 & 1.5175 \\
\hline 4,015 & 0.19 & 0.2 & 0.64 & 0.31 & 0.49 & 0.39 & 60.95 & 417 & 1.05 & 19.05 & nd & 1.0833 & 1.3925 \\
\hline 4,070 & 0.22 & 0.39 & 0.6 & 0.65 & 0.36 & 0.61 & 61.22 & 379 & 0.98 & 39.8 & nd & 1.0367 & 1.6867 \\
\hline 4,088 & 0.15 & 0.3 & 0.34 & 0.88 & 0.33 & 0.45 & 53.13 & 352 & 0.64 & 46.88 & nd & 1.11 & 1.5471 \\
\hline 4,130 & 0.16 & 0.16 & 0.48 & 0.33 & 0.5 & 0.32 & 63.16 & 360 & 0.76 & 21.05 & nd & 1.0425 & 1.36 \\
\hline 4,195 & 0.23 & 0.28 & 0.27 & 1.04 & 0.45 & 0.51 & 15.25 & 362 & 1.77 & 15.82 & nd & 0.9967 & nd \\
\hline $4,200.5$ & 0.19 & 0.27 & 0.15 & 1.8 & 0.41 & 0.46 & 24.19 & 395 & 0.62 & 43.55 & nd & 1.1375 & 1.275 \\
\hline 4,220 & 0.31 & 0.33 & 0.33 & 1 & 0.48 & 0.64 & 11.46 & 458 & 2.88 & 11.46 & nd & nd & 1.47 \\
\hline 4,250 & 0.57 & 0.63 & 0.28 & 2.25 & 0.48 & 1.2 & 21.54 & 366 & 1.3 & 48.46 & nd & 1.1725 & 1.5317 \\
\hline 4,320 & 0.29 & 0.69 & 0.29 & 2.38 & 0.3 & 0.98 & 16.86 & 366 & 1.72 & 40.12 & 1.03 & 1.1743 & nd \\
\hline 4,380 & $\mathrm{nr}$ & $\mathrm{nr}$ & $\mathrm{nr}$ & $\mathrm{nr}$ & $\mathrm{nr}$ & $\mathrm{nr}$ & $\mathrm{nr}$ & $\mathrm{nr}$ & $\mathrm{nr}$ & $\mathrm{nr}$ & 1.025 & 1.1618 & nd \\
\hline 4,495 & $\mathrm{nr}$ & $\mathrm{nr}$ & $\mathrm{nr}$ & $\mathrm{nr}$ & $\mathrm{nr}$ & $\mathrm{nr}$ & $\mathrm{nr}$ & $\mathrm{nr}$ & $\mathrm{nr}$ & $\mathrm{nr}$ & 1.034 & 1.1984 & nd \\
\hline 4,630 & $\mathrm{nr}$ & $\mathrm{nr}$ & $\mathrm{nr}$ & $\mathrm{nr}$ & $\mathrm{nr}$ & $\mathrm{nr}$ & $\mathrm{nr}$ & $\mathrm{nr}$ & $\mathrm{nr}$ & $\mathrm{nr}$ & 1.0767 & 1.1956 & 1.4975 \\
\hline 4,740 & $\mathrm{nr}$ & $\mathrm{nr}$ & $\mathrm{nr}$ & $\mathrm{nr}$ & $\mathrm{nr}$ & $\mathrm{nr}$ & $\mathrm{nr}$ & $\mathrm{nr}$ & $\mathrm{nr}$ & $\mathrm{nr}$ & 1.0325 & 1.23 & 1.6025 \\
\hline 4,795 & $\mathrm{nr}$ & $\mathrm{nr}$ & $\mathrm{nr}$ & $\mathrm{nr}$ & $\mathrm{nr}$ & $\mathrm{nr}$ & $\mathrm{nr}$ & $\mathrm{nr}$ & $\mathrm{nr}$ & $\mathrm{nr}$ & 0.9833 & 1.1567 & nd \\
\hline 4,900 & $\mathrm{nr}$ & $\mathrm{nr}$ & $\mathrm{nr}$ & $\mathrm{nr}$ & $\mathrm{nr}$ & $\mathrm{nr}$ & $\mathrm{nr}$ & $\mathrm{nr}$ & $\mathrm{nr}$ & $\mathrm{nr}$ & nd & nd & nd \\
\hline 4,970 & $\mathrm{nr}$ & $\mathrm{nr}$ & $\mathrm{nr}$ & $\mathrm{nr}$ & $\mathrm{nr}$ & $\mathrm{nr}$ & $\mathrm{nr}$ & $\mathrm{nr}$ & $\mathrm{nr}$ & $\mathrm{nr}$ & 0.3981 & nd & nd \\
\hline 2,385 & 3.44 & 11.2 & 4.32 & 2.59 & 0.24 & 14.64 & 91.72 & 428 & 4.71 & 237.79 & $\mathrm{nr}$ & $\mathrm{nr}$ & $\mathrm{nr}$ \\
\hline 2,405 & 2.8 & 10 & 7.3 & 1.37 & 0.22 & 12.8 & 119.87 & 431 & 6.09 & 164.2 & $\mathrm{nr}$ & $\mathrm{nr}$ & $\mathrm{nr}$ \\
\hline 2,769 & nd & nd & nd & nd & nd & nd & nd & nd & 0.1 & nd & $\mathrm{nr}$ & $\mathrm{nr}$ & $\mathrm{nr}$ \\
\hline 2,835 & 2.36 & 3.19 & 1.9 & 1.68 & 0.43 & 5.55 & 263.89 & 370 & 0.72 & 443.06 & $\mathrm{nr}$ & $\mathrm{nr}$ & $\mathrm{nr}$ \\
\hline 3,391 & nd & nd & nd & nd & nd & nd & nd & nd & 0.23 & nd & $\mathrm{nr}$ & $\mathrm{nr}$ & $\mathrm{nr}$ \\
\hline $3,538.5$ & 2.18 & 1.62 & 0.96 & 1.69 & 0.57 & 3.8 & 73.28 & 451 & 1.31 & 123.66 & $\mathrm{nr}$ & $\mathrm{nr}$ & $\mathrm{nr}$ \\
\hline $4,244.5$ & 18.92 & 2.92 & 0.72 & 4.06 & 0.87 & 21.84 & 76.6 & 436 & 0.94 & 310.64 & $\mathrm{nr}$ & $\mathrm{nr}$ & $\mathrm{nr}$ \\
\hline $4,275.4$ & 3.15 & 0.93 & 0.18 & 5.17 & 0.77 & 4.08 & 15.38 & 362 & 1.17 & 79.49 & $\mathrm{nr}$ & $\mathrm{nr}$ & $\mathrm{nr}$ \\
\hline 4,278 & 31.12 & 2.58 & 0.41 & 6.29 & 0.92 & 33.7 & 48.81 & 433 & 0.84 & 307.14 & $\mathrm{nr}$ & $\mathrm{nr}$ & $\mathrm{nr}$ \\
\hline $4,281.5$ & 4.57 & 0.99 & 0.17 & 5.82 & 0.82 & 5.56 & 13.28 & 420 & 1.28 & 77.34 & $\mathrm{nr}$ & $\mathrm{nr}$ & $\mathrm{nr}$ \\
\hline $4,474.5$ & 24.6 & 1.45 & 0.38 & 3.82 & 0.94 & 26.05 & 49.35 & 429 & 0.77 & 188.31 & $\mathrm{nr}$ & $\mathrm{nr}$ & $\mathrm{nr}$ \\
\hline $4,486.2$ & nd & nd & nd & nd & nd & nd & nd & nd & 0.19 & nd & $\mathrm{nr}$ & $\mathrm{nr}$ & $\mathrm{nr}$ \\
\hline 4,487 & 23.44 & 1.27 & 0.46 & 2.76 & 0.95 & 24.71 & 65.71 & 428 & 0.7 & 181.43 & $\mathrm{nr}$ & $\mathrm{nr}$ & $\mathrm{nr}$ \\
\hline $4,488.9$ & 0.9 & 0.47 & 0.06 & 7.83 & 0.66 & 1.37 & 13.04 & 360 & 0.46 & 102.17 & $\mathrm{nr}$ & $\mathrm{nr}$ & $\mathrm{nr}$ \\
\hline $4,500.9$ & nd & nd & nd & nd & nd & nd & nd & nd & 0.42 & nd & $\mathrm{nr}$ & $\mathrm{nr}$ & $\mathrm{nr}$ \\
\hline $4,655.5$ & nd & nd & nd & nd & nd & nd & nd & nd & 0.22 & nd & $\mathrm{nr}$ & $\mathrm{nr}$ & $\mathrm{nr}$ \\
\hline $4,659.9$ & 4.67 & 0.34 & 0.14 & 2.43 & 0.93 & 5.01 & 32.56 & 355 & 0.43 & 79.07 & $\mathrm{nr}$ & $\mathrm{nr}$ & $\mathrm{nr}$ \\
\hline $4,663.5$ & nd & nd & nd & nd & nd & nd & nd & nd & nd & nd & $\mathrm{nr}$ & $\mathrm{nr}$ & $\mathrm{nr}$ \\
\hline
\end{tabular}


Table 1 continued

\begin{tabular}{|c|c|c|c|c|c|c|c|c|c|c|c|c|c|}
\hline \multirow[t]{2}{*}{ Depth } & \multirow[t]{2}{*}{$S_{1}$} & \multirow[t]{2}{*}{$S_{2}$} & \multirow[t]{2}{*}{$S_{3}$} & \multirow[t]{2}{*}{$S_{2} / S_{3}$} & \multirow[t]{2}{*}{ PI } & \multirow[t]{2}{*}{$S_{1}+S_{2}$} & \multirow[t]{2}{*}{ OI } & \multirow[t]{2}{*}{$T_{\max }$} & \multirow[t]{2}{*}{ TOC } & \multirow[t]{2}{*}{$\mathrm{HI}$} & \multicolumn{3}{|l|}{$R_{\mathrm{o}}$} \\
\hline & & & & & & & & & & & $\begin{array}{l}\text { Caved } \\
\text { population }\end{array}$ & Indigenous & $\begin{array}{l}\text { Oxidized } \\
\text { population }\end{array}$ \\
\hline $4,742.5$ & nd & nd & nd & nd & nd & nd & nd & nd & nd & nd & $\mathrm{nr}$ & $\mathrm{nr}$ & $\mathrm{nr}$ \\
\hline $4,879.9$ & nd & nd & nd & nd & nd & nd & nd & nd & 0.15 & nd & $\mathrm{nr}$ & $\mathrm{nr}$ & $\mathrm{nr}$ \\
\hline $4,889.5$ & nd & nd & nd & nd & nd & nd & nd & nd & nd & nd & $\mathrm{nr}$ & $\mathrm{nr}$ & $\mathrm{nr}$ \\
\hline $4,906.8$ & nd & nd & nd & nd & nd & nd & nd & nd & 0.23 & nd & $\mathrm{nr}$ & $\mathrm{nr}$ & $\mathrm{nr}$ \\
\hline $4,928.6$ & nd & nd & nd & nd & nd & nd & nd & nd & 0.06 & nd & $\mathrm{nr}$ & $\mathrm{nr}$ & $\mathrm{nr}$ \\
\hline $4,987.5$ & 21.52 & 0.57 & 0.26 & 2.19 & 0.97 & 22.09 & 18.84 & 385 & 1.38 & 41.3 & $\mathrm{nr}$ & $\mathrm{nr}$ & $\mathrm{nr}$ \\
\hline 5,195 & nd & nd & nd & nd & nd & nd & nd & nd & nd & nd & $\mathrm{nr}$ & $\mathrm{nr}$ & $\mathrm{nr}$ \\
\hline 5,292 & 38.16 & 1.2 & 0.17 & 7.06 & 0.97 & 39.36 & 12.69 & 424 & 1.34 & 89.55 & $\mathrm{nr}$ & $\mathrm{nr}$ & $\mathrm{nr}$ \\
\hline $5,310.9$ & nd & nd & nd & nd & nd & nd & nd & nd & nd & nd & $\mathrm{nr}$ & $\mathrm{nr}$ & $\mathrm{nr}$ \\
\hline $5,333.3$ & 6.01 & 0 & 24.64 & 0 & 1 & 6.01 & 1811.76 & nd & 1.36 & 0 & $\mathrm{nr}$ & $\mathrm{nr}$ & $\mathrm{nr}$ \\
\hline $5,351.3$ & nd & nd & nd & nd & nd & nd & nd & nd & 0.44 & nd & $\mathrm{nr}$ & $\mathrm{nr}$ & $\mathrm{nr}$ \\
\hline $5,372.5$ & nd & nd & nd & nd & nd & nd & nd & nd & nd & nd & $\mathrm{nr}$ & $\mathrm{nr}$ & $\mathrm{nr}$ \\
\hline 5,407 & 16.6 & 0.26 & 0.12 & 2.17 & 0.98 & 16.86 & 13.64 & 385 & 0.88 & 29.55 & $\mathrm{nr}$ & $\mathrm{nr}$ & $\mathrm{nr}$ \\
\hline 5,437 & 29.24 & 3.38 & 0.48 & 7.04 & 0.9 & 32.62 & 40 & 431 & 1.2 & 281.67 & $\mathrm{nr}$ & $\mathrm{nr}$ & $\mathrm{nr}$ \\
\hline $5,447.5$ & nd & nd & nd & nd & nd & nd & nd & nd & 0.1 & nd & $\mathrm{nr}$ & $\mathrm{nr}$ & $\mathrm{nr}$ \\
\hline $5,508.5$ & nd & nd & nd & nd & nd & nd & nd & nd & nd & nd & $\mathrm{nr}$ & $\mathrm{nr}$ & $\mathrm{nr}$ \\
\hline 5,628 & 17.8 & 0.61 & 1.32 & 0.46 & 0.97 & 18.41 & 118.92 & 425 & 1.11 & 54.96 & $\mathrm{nr}$ & $\mathrm{nr}$ & $\mathrm{nr}$ \\
\hline $5,712.5$ & 29.6 & 8.8 & 0.2 & 44 & 0.77 & 38.4 & 0.47 & 429 & 42.35 & 20.78 & $\mathrm{nr}$ & $\mathrm{nr}$ & $\mathrm{nr}$ \\
\hline $5,717.5$ & 25.2 & 3.25 & 0.28 & 11.61 & 0.89 & 28.45 & 7.55 & 385 & 3.71 & 87.6 & $\mathrm{nr}$ & $\mathrm{nr}$ & $\mathrm{nr}$ \\
\hline $4,796.7$ & 0.91 & 0.86 & 0.18 & 4.78 & 0.51 & 1.77 & 50.28 & 358 & 0.36 & 240.22 & $\mathrm{nr}$ & $\mathrm{nr}$ & $\mathrm{nr}$ \\
\hline $4,790.5$ & 0.31 & 0.24 & 0.14 & 1.71 & 0.57 & 0.55 & 28.69 & 347 & 0.49 & 49.18 & $\mathrm{nr}$ & $\mathrm{nr}$ & $\mathrm{nr}$ \\
\hline $5,005.6$ & 9.3 & 0.75 & 0.44 & 1.7 & 0.93 & 10.05 & 139.68 & 422 & 0.32 & 238.1 & $\mathrm{nr}$ & $\mathrm{nr}$ & $\mathrm{nr}$ \\
\hline
\end{tabular}

Depth in mTVD unless and until mentioned otherwise; $S_{1}, S_{2}, \mathrm{~S}_{3}$ in $\mathrm{mg} \mathrm{HC} / \mathrm{g}$ rock; $T_{\max }$ in ${ }^{\circ} \mathrm{C}$; TOC in wt $\% ; R_{\mathrm{o}}$ in $\%$

$P I$ production index, $O I$ oxygen index in $\mathrm{mg} \mathrm{HC/g}$ organic carbon, $H I$ hydrogen index in $\mathrm{mg} \mathrm{HC} / \mathrm{g}$ organic carbon, $n r$ not recorded, $n d$ not detected

Index as a function of temp, oxygen index, TOC, etc. Plotting $T_{\max }$ versus $\mathrm{HI}$ shows the different maturation paths of different kerogen types (Fig. 5). $T_{\max }$ values correlate directly with vitrinite reflectance values. The type and maturity of organic matter in petroleum source rocks have been characterized from rock-eval pyrolysis data (Fig. 6). Geochemical parameters including HI [hydrogen index, $\left(S_{2} /\right.$ TOC $) \times 100$ ], OI [oxygen index, $\left(S_{3} /\right.$ TOC) $\times 100$ ] and PI [production index, $S_{1} /\left(S_{1}+S_{2}\right)$ ] are calculated. $S_{1}$ versus TOC plot gives a clear indication of the indigenous hydrocarbons and non-indigenous hydrocarbons (Fig. 7). Pyrolysis experiments were repeated for some samples at early sample weight to ensure that the rock-eval/TOC detector was not overloaded by generated hydrocarbons during the original runs (Ibrahimbas and Riediger 2002).

The analytical procedure, discussion and details of rockeval pyrolysis parameters are available in Peters (1986) and Snowdon et al. (1998). Table 2 provides an interpretive guidelines based on $S_{2}$ values from rock-eval pyrolysis. Accordingly, the present study objectives are aimed to evaluate the hydrocarbon source potential from the available core sample data and to study the thermal maturity of the geological sequence in parts of $\mathrm{KG}$ basin.

Source facies and TOC

Geochemical data reveal that interval 2,325-2,420 m (Early Eocene) TOC contents recorded are good to very good (1.64-2.59 wt \%). Rock-eval pyrolysis data reveal moderate potential yields $\left(S_{1}+S_{2}=2.99-4.87 \mathrm{mg} \mathrm{HC} / \mathrm{g}\right.$ rock). Figure 9 indicates a fair hydrocarbon source potential. Hydrogen indices were recorded (171-178) implying the presence of predominantly humic gas-prone kerogen (Type-III). Rock-eval pyrolysis reveals an average potential yield $\left(S_{1}+S_{2}=1.09 \mathrm{mg} \mathrm{HC} / \mathrm{g}\right.$ rock $)$ and a low hydrogen index (=128 mg HC/g organic carbon) (Figs. 6, 8). The data suggest the sample has fair hydrocarbon source potential and contain predominantly gas-prone kerogen and has been cross-checked with well-testing reports (Kaawache and Mahapatra 2005). Visual kerogen composition data confirm the presence of predominantly of 


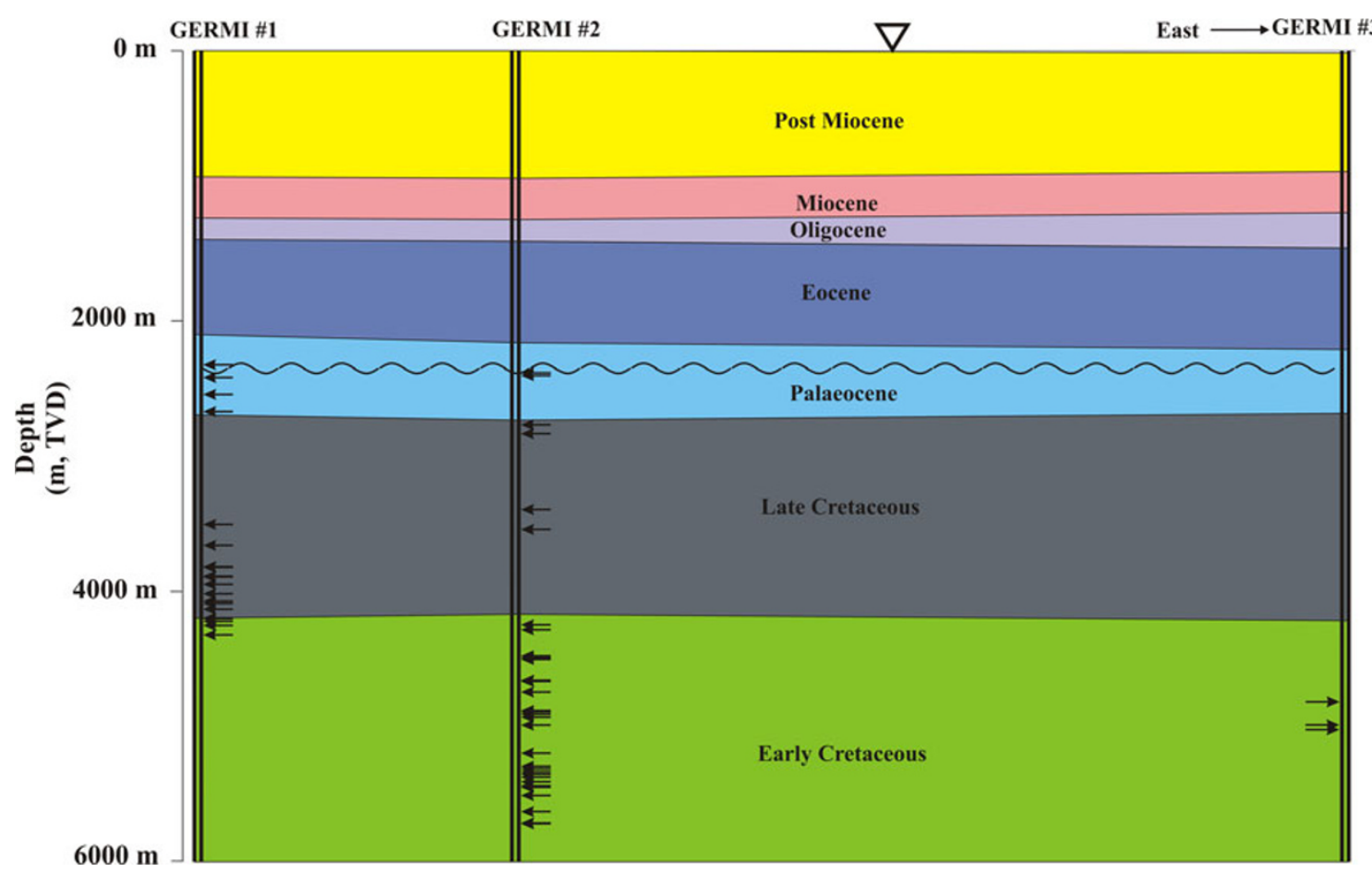

Fig. 4 Stratigraphy and lithology depth profile for well bores (GERMI \#1, GERMI \#2 and GERMI \#3). Arrows indicate the sample locations

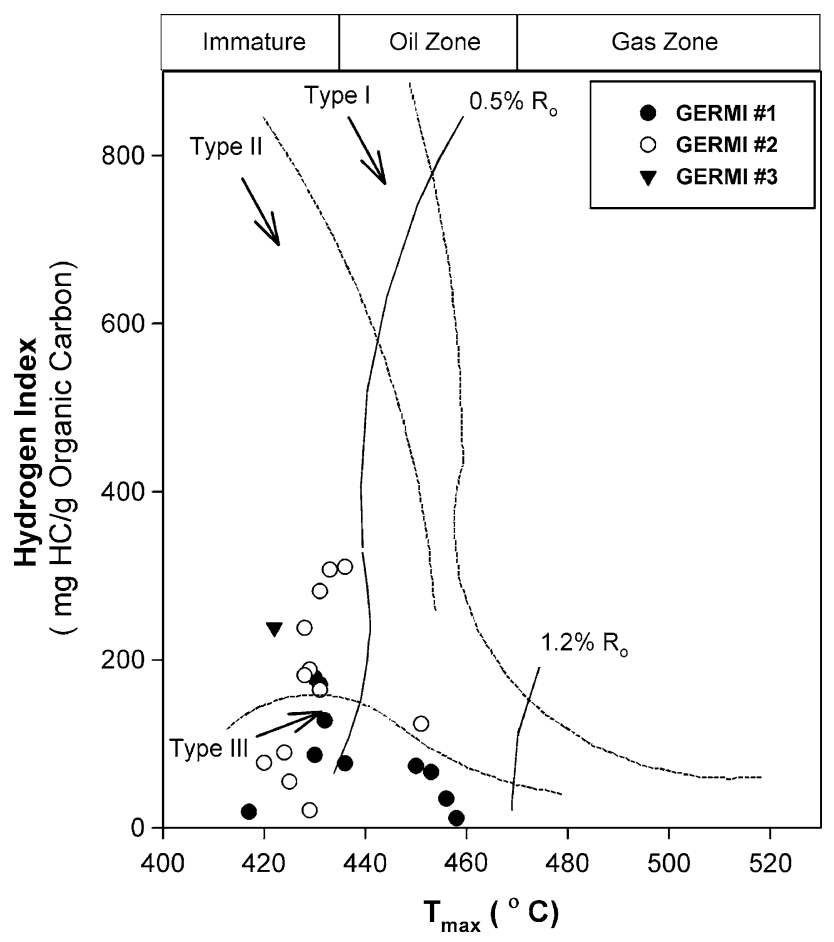

Fig. 5 Cross plot of rock-eval $T_{\max }$ versus hydrogen index (HI) data showing the range in thermal maturity as well as hydrocarbon generative (kerogen) types of samples, indicating immature to mature type-III kerogen from GERMI \#1 and GERMI \#2 while immature samples from GERMI \#3

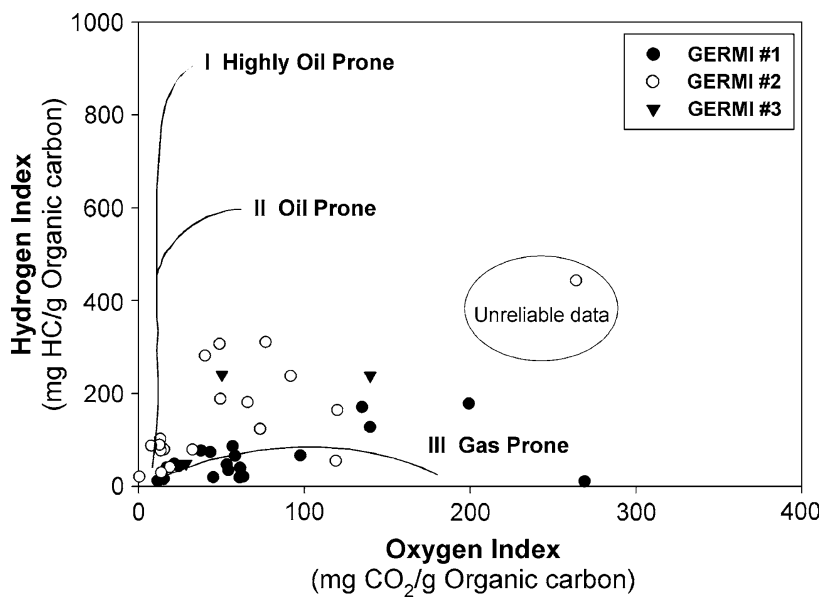

Fig. 6 HI versus OI plots on the modified Van Krevelen diagram of samples, indicating type-III gas-prone samples of all the borings

non-fluorescent kerogen (58\%) and vitrinite particles/ Type-III kerogens (30\%) (Fig. 6).

Hydrogen indices (10-87 mg HC/g organic carbon) suggest the presence of gas-prone kerogen (Type-III). This is also confirmed by visual kerogen typing analysis, which was performed on all samples interval 4,070-5,050 m (Early Cretaceous) comprise mainly medium dark grey to olive grey claystones and medium dark grey to dark grey shales. TOC contents for these samples range from 0.20 to 


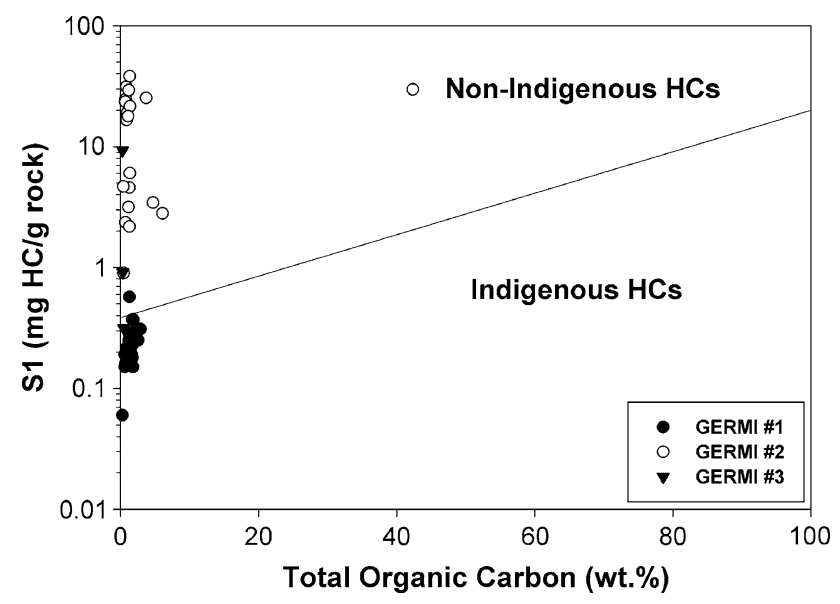

Fig. $7 \quad S_{1}$ versus TOC plots of samples indicating indigenous hydrocarbons of GERMI \#1 and non-indigenous hydrocarbons of GERMI \#2 and GERMI \#3, except one sample (adapted from Hunt 1995)

Table 2 Standard criteria for rating potential source rocks based on $S_{2}$ values after Ibrahimbas and Riediger 2002

\begin{tabular}{ll}
\hline Rating & $S_{2}(\mathrm{mg} \mathrm{HC} / \mathrm{g}$ rock $)$ \\
\hline Poor & $<2.00$ \\
Fair & $2.00-5.00$ \\
Good & $>5.00$ \\
\hline
\end{tabular}

$2.88 \mathrm{wt} \%$. Rock-eval pyrolysis analysis reveal low to moderate hydrogen indices (ranging from 11 to $185 \mathrm{mg}$ $\mathrm{HC} / \mathrm{g}$ organic carbon) implying the presence of predominantly gas-prone kerogen (Type-III) (Fig. 8). Visual kerogen typing analysis performed on these samples show the abundance of non-fluorescent amorphous debris (10-73\%), with secondary amounts of vitrinite particles (7-56\%). Toward the base the kerogen are dominated by vitrinite, semifusinite and inertinite maceral.

In GERMI \#2 the conventional core samples from the depth range of $2,385-5,718 \mathrm{~m}$ are taken for TOC determination. Overall, the samples contain relatively low TOC $(<2 \%)$ indicative of poor to moderate organic richness. However, the low TOC contents suggest the section has little potential for hydrocarbon generation (Fig. 8). The rock-eval pyrolysis indicates the potential source to be gas prone at best. $S_{1}+S_{2}$ versus TOC plots of samples indicating fair to good source rock potential of samples from GERMI \#2 (Fig. 9). Based on $T_{\max }$ values between 370 and $431{ }^{\circ} \mathrm{C}$, the Late Claystone section appears to be immature to marginally mature (Fig. 9). The early value of $T_{\max }$ $370{ }^{\circ} \mathrm{C}$ could be due to the presence of free hydrocarbons. Hydrogen indices (HI) ( 164 to $443 \mathrm{mg} \mathrm{HC/g}$ organic carbon) and suggest that the samples are oil prone (Fig. 5). Between 3,538.5 and $5,717.5 \mathrm{~m}$ the TOC value ranges from $0.1 \%(5,447.5 \mathrm{~m})$ to $3.71 \%(5,717.5 \mathrm{~m})$, with most

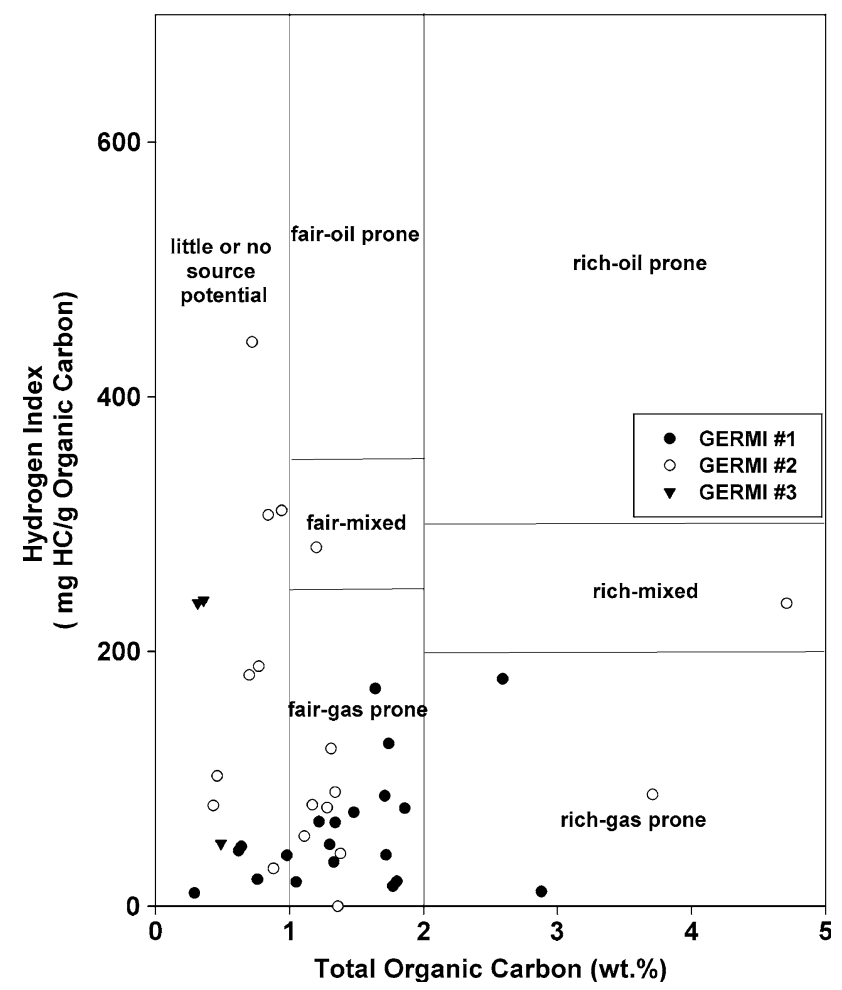

Fig. 8 HI versus TOC plots of samples indicating fair to rich gas potential of samples from GERMI \#1 and GERMI \#2 while little or no potential from GERMI \#3

values between 0.4 and $1.5 \%$ (fair to good) except some higher TOC values at $5,712.5 \mathrm{~m}$, which is because of presence of coal. $S_{1}$ values ranges from 2.18 to $29.6 \mathrm{mg}$ $\mathrm{HC} / \mathrm{g}$ rock characterizing excellent levels of free hydrocarbons, except $0.9 \mathrm{mg} / \mathrm{g}$ value at 4,488.9 m. Hydrocarbon generating potentials are poor to moderate $\left(S_{2}\right.$ values between 0.26 and $3.38 \mathrm{mg} \mathrm{HC/g}$ rock) and $T_{\max }$ values between around 420 and $450{ }^{\circ} \mathrm{C}$ reflect immaturity to maturity (Fig. 9). Low $T_{\max }$ values below $400{ }^{\circ} \mathrm{C}$ are likely to be artifact, caused by $T_{\max }$ suppression due to the presence of considerable level of free hydrocarbons $\left(S_{1}\right)$. HI vary between $30 \mathrm{mg} \mathrm{HC/g}$ organic carbon (gas) and 311 (gas and oil), with most values indicating a more gas-prone character of the source sediments than the oil. Interpreted hydrocarbons are matching with well-testing reports (Asianto and Losier 2007; Asianto and Rosier 2007).

The number of samples from GERMI \#3 are very limited (Fig. 4) but still those sample were showing $\mathrm{HI}$ in the range of $32-50 \mathrm{mg} \mathrm{HC/g}$ organic carbon with most value indicating the gas-prone character of the sediments. The hydrocarbon generation potential varies from poor to moderate (Fig. 6). The obtained geochemical results have been confirmed from well-testing reports (Maclennan et al. 2007; Jong and Pelser 2007). Due to side tracking borehole GERMI \#3 has got three samples for which geochemical analysis has been carried out.

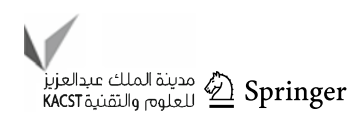


Fig. $9 S_{1}+S_{2}$ versus TOC plots of samples indicating fair to good source rock potential of samples from GERMI \#1 and GERMI \#2 while poor potential from GERMI \#3

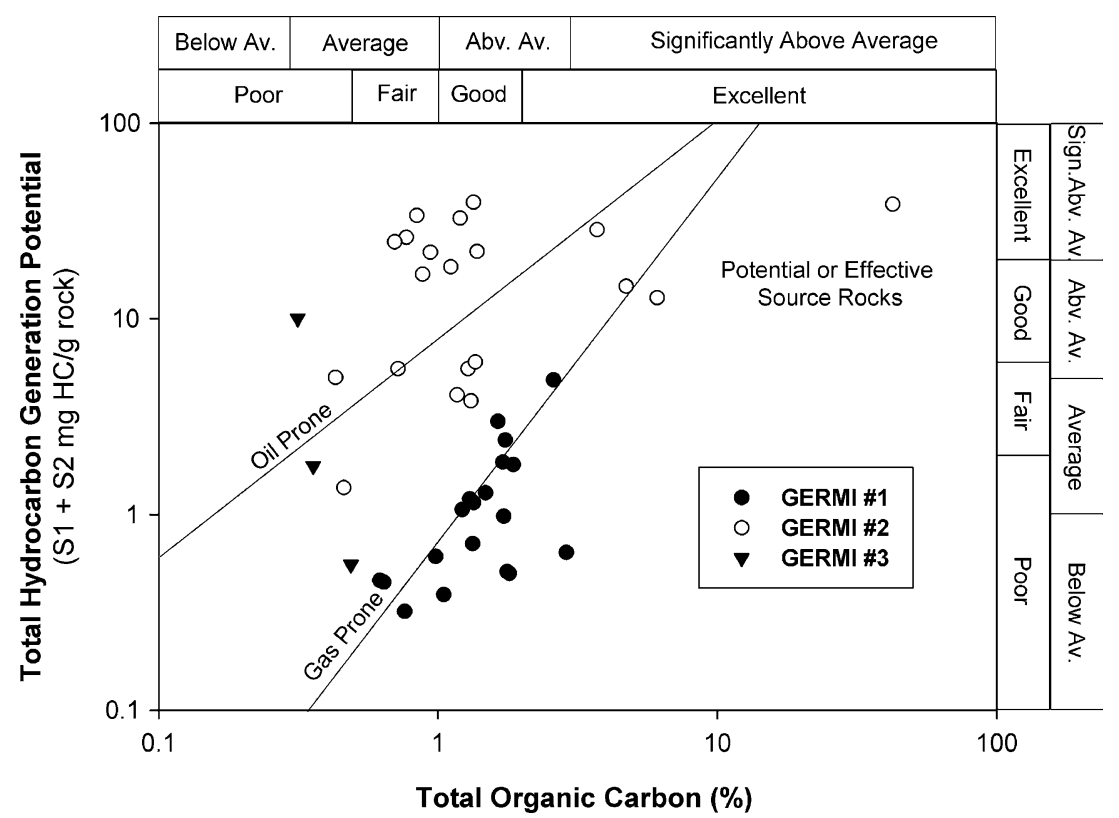

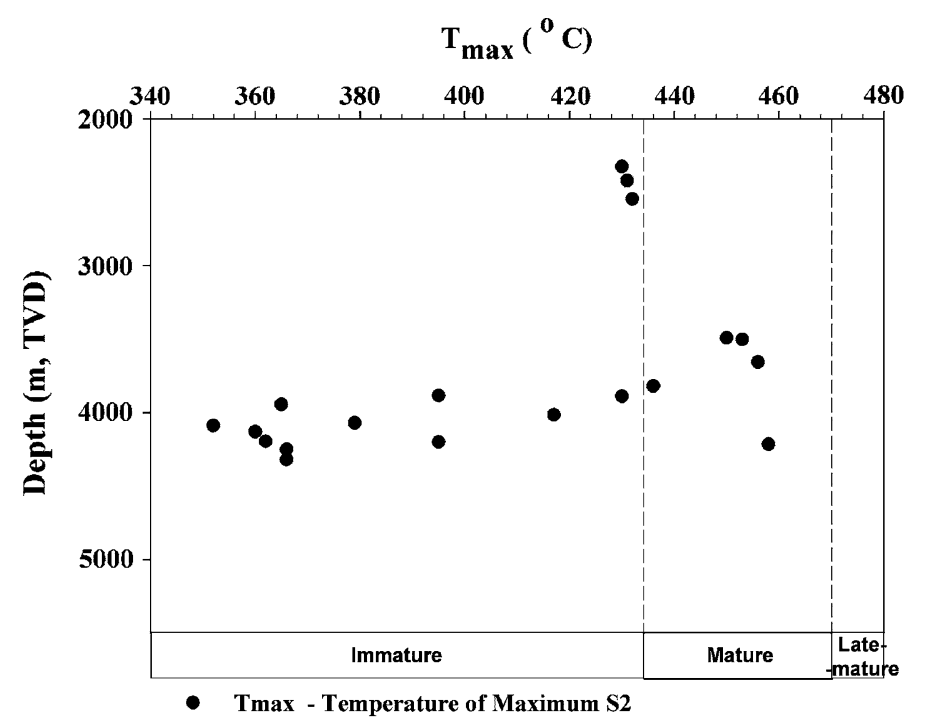

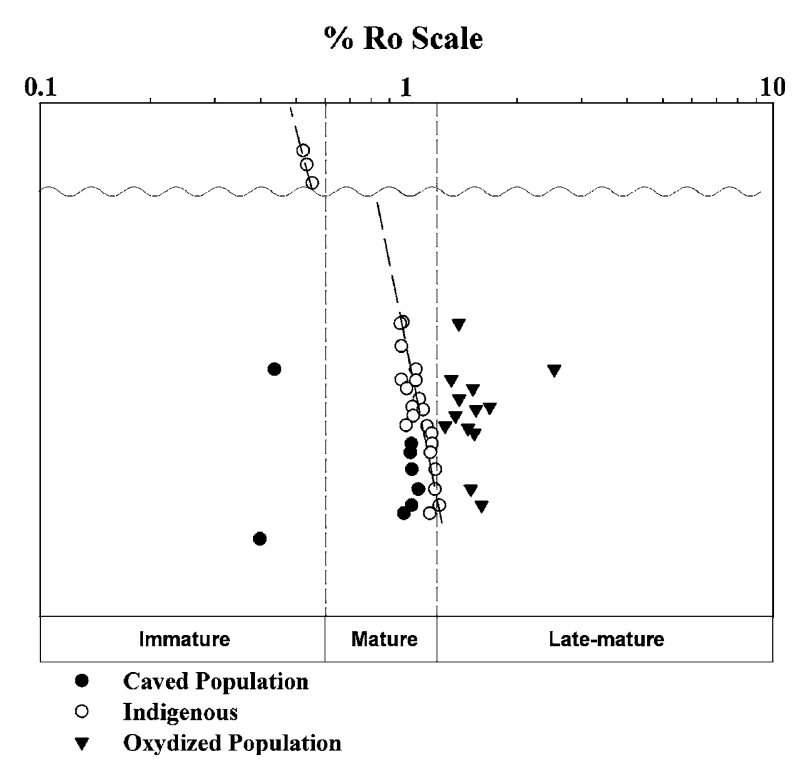

Fig. $10 T_{\max }$ and $R_{\mathrm{o}}$ depth profile for GERMI \#1 indicating break in maturity profile due to significant erosion during the uplifted prior to deposition of overlying formations. Note the matured indigenous core samples

Maturity evaluation, vitrinite reflectivity analysis

In GERMI \#1 a total of 30 samples throughout the interval 2,325-5,050 m were submitted for vitrinite reflectivity analysis (polished whole rock). The results are generally fair to good based on variably low to abundant recoveries of vitrinite particles. Samples from depth 2,672, 4,900, 4,995, 5,010 and $5,050 \mathrm{~m}$ were found to be barren of indigenous vitrinite particles (Asianto and Losier 2007; Asianto and Rosier 2007). Recorded mean vitrinite reflectivity values range from $0.52 \%$ $R_{\mathrm{O}}$ (at $2,325 \mathrm{~m}$ ) to $0.55 \% R_{\mathrm{O}}$ (at $2,545 \mathrm{~m}$ ) (Fig. 10). The interpreted vitrinite reflectivity profile shows that the section is marginally mature for oil generation. Below $3,492 \mathrm{~m}$ there is a rapid increase in vitrinite reflectivity values from late mature to post mature. The $R_{\mathrm{o}}$ values increase uniformly from $0.98 \% R_{\mathrm{o}}$ (at 3,492 m) to $1.16 \% R_{\mathrm{o}}$ (at $4,795 \mathrm{~m}$ ) indicating that the section is late mature for oil generation (Fig. 10). As mentioned earlier, sample 2,672 $\mathrm{m}$ was found barren of indigenous vitrinite particles. It is noted however, that spore colouration analysis on this sample (discussed below) confirms it is marginal mature for oil generation. Hence, the rapid increase in vitrinite reflectivity within the interval $2,673-3,492 \mathrm{~m}$ is thought to be associated with a significant unconformity which occurs at depth c. $2,675 \mathrm{~m}$. 
The argillaceous sediments analyzed throughout the sequence (interval 2,325-5,050 m, Early Eocene to Early Cretaceous) display variably very low to good organic richness (TOC in the range of $0.20-2.88 \mathrm{wt} \%$ ). These sediments are considered to have poor to fair hydrocarbon source potential $\left(S_{1}+S_{2}=0.09-4.87 \mathrm{mg} \mathrm{HC} / \mathrm{g}\right.$ rock) and contain mainly gas-prone kerogen based on low to moderate $\mathrm{HI}$ values (11-185 $\mathrm{mg} \mathrm{HC/g}$ organic carbon). Visual kerogen analysis on these samples confirms the presence of predominantly gas-prone kerogen (90-99\%). The gasprone kerogen consists of vitrinite maceral and non-fluorescent amorphous debris. The non-fluorescent amorphous material is possibly sapropelic debris which has been affected by thermal maturity.

\section{Conclusions}

Based on the detailed analysis of the rock samples from three different deep offshore wells, the following major conclusion can be drawn.

- A break in maturity profile observed in GERMI \#1 suggests that there is a significant amount of erosion. This area is known to be tectonically active; it can be interpreted that the erosion might have taken place during the uplifted period prior to deposition of overlying formation. Additionally, reported high heat flow values have been contributed significantly for the maturity levels. From the extrapolation of $R_{\mathrm{o}}$ profile to the surface indicates that the reaction might be at a much deeper depth of burial and have experienced high temperature. There could be alternate interpretation of the break in maturity profile of the well. However, additional data like FAMM (fluorescence alteration of multiple macerals), VIRF (vitrinite inertinite reflectance and fluorescence) and burial history modeling are required.

- $S_{1}$ versus TOC plot indicates possible indigenous hydrocarbons from borehole GERMI \#1 while nonindigenous hydrocarbons from boreholes GERMI \#2 and GERMI \#3.

- Different rock-eval pyrolysis parameters are suggestive of immature to early mature gas-prone shales from borehole GERMI \#1 while early mature to mature oil and gas-prone shales from borehole GERMI \#2. Limited samples from GERMI \#3 are indicative of immature shales. Well-testing analysis also supports the type of hydrocarbons interpreted.

Acknowledgments We would like to express our deep gratitude to Gujarat State Petroleum Corporation Ltd. (GSPC) for their support and permission in getting this paper published. We are also grateful to the staff of GERMI and GSPC for sharing their interpretation insights throughout several brainstorming sessions. Thanks are also due to those who helped in their own way to get this study completed.

Open Access This article is distributed under the terms of the Creative Commons Attribution License which permits any use, distribution, and reproduction in any medium, provided the original author(s) and the source are credited.

\section{References}

Asianto A, Losier C (2007) WCP testing report. Gujarat State Petroleum Corporation Limited (unpublished)

Asianto A, Rosier C (2007) WCP testing report. Gujarat State Petroleum Corporation Limited (unpublished)

Behar F, Beaumont V, Penteako HLD (2001) Rock-eval 6 technology: performances and developments. Revue de L'institut Francais du Petrole 56(2):111-134

Biswas SK (1998) Overview of sedimentary basins of India and their hydrocarbon resource potential. In: Proceedings National Symposium on Recent Researches in Sedimentary Basins. BHU, Varanasi, pp 1-25

Govindan A (1984) Stratigraphy and sedimentation of East-Godavari sub-basin. Pet Asia J 7(1):132-146

Gupta SK (2006) Basin architecture and petroleum system of Krishna Godavari basin, east coast of India. The Leading Edge, pp 830-837. doi:10.1190/1.2221360

Hunt JM (1995) Petroleum geochemistry and geology, 2nd edn. W. H. Freeman and Company, New York, p 743

Ibrahimbas A, Riediger C (2002) Hydrocarbon source rock potential as determined by rock-eval 6/TOC pyrolysis. http://www.em. gov.bc.ca/Mining/Geoscience/PublicationsCatalogue/OilGas/OG Reports/Documents/2004/OG_Report2004_ibrahimbas_riediger. pdf

IHS Energy (2005) Basin monitor, Krishna-Godavari basin, India (unpublished)

Jong PD, Pelser G (2007) WCP testing report. Gujarat State Petroleum Corporation Limited (unpublished)

Kaawache S, Mahapatra B (2005) WCP testing report. Gujarat State Petroleum Corporation Limited (unpublished)

Kaila KL, Murty PRK, Rao VK, Venkateswarlu N (2005) Deep seismic sounding in the Godavari Graben and Godavari (coastal) basin, India. Tectonophysics 173(1):307-317

Kumar SP (1983) Geology and hydrocarbon prospects of KrishnaGodavari and Cauvery basins. Pet Asia J 8(1):57-65

Lafargue E, Marquis F, Pillot D (1998) Rock-eval 6 applications in hydrocarbon exploration, production, and soil contamination studies. Revue de L'institut Francais du Petrole 53(4):421-437. doi: $10.2516 /$ ogst: 1998036

Maclennan J, Pelser G, Gangl J (2007) WCP testing report. Gujarat State Petroleum Corporation Limited (unpublished)

Murthy KSR, Subrahmanyam AS, Lakshminarayana S et al (1995) Some geodynamic aspects of the Krishna-Godavari basin, east coast of India. Cont Shelf Res 15(7):779-785. doi:10.1016/ 0278-4343(94)00044-N

Murthy KSR, Subrahmanyam V, Subrahmanyam AS et al. (2010) Land-Ocean Tectonics (LOTs) and the associated seismic hazard over the Eastern Continental Margin of India (ECMI). Nat Hazards 55(2):167-175. https://10.0.3.239/s11069-010-9523-8

Peters KE (1986) Guidelines for evaluating petroleum source rocks using programmed pyrolysis. Am Assoc Pet Geol Bull 70(3): 318-329

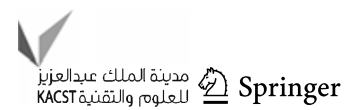


Rai A, Chandrasekhar P, Mishra VN (1998) Indian sedimentary basins and their hydrocarbon potential. In: Proceedings National Symposium on Recent Researches in Sedimentary Basins. BHU, Varanasi, pp 91-103

Rao GN (2001) Sedimentation, stratigraphy and petroleum potential of Krishna-Godavari basin, East Coast of India. Am Assoc Pet Geol Bull 85(9):1623-1643

Saxena K, Raina A, Pandey HC et al. (2011) Application of compound specific isotopic analysis to fine tune oil-oil, oilsource correlations in KG basin, India. The Second South Asian Geoscience Conference and Exhibition, GEO India 636:1-7. http://apgindia.org/pdf/636.pdf

Snowdon LR, Fowler MG, Riediger CL (1998) Interpretation of organic geochemical data. Short Course Notes, CSPG, Calgary
Subrahmanyam V, Subrahmanyam AS, Murty GPS et al (2008) Morphology and tectonics of Mahanadi basin, northeastern margin of India from geophysical studies. Mar Geol 253:63-72

Tidar ABN, Budi H (2005) Petroleum geochemistry, KrishnaGodavari basin, offshore east coast of India. Technical report, Gujarat State Petroleum Corporation Limited (unpublished)

Tissot BP, Welte DH (1978) Petroleum formation and occurrence. Springer, Berlin

Venkatarangan R, Ray D (1993) Geology and petroleum systems, Krishna-Godavari basin. In: Biswas SK et al (ed) Proceedings of the Second Seminar on Petroliferous Basins of India. Ind Petrol Publ, Dehradun 1:331-354 